\title{
Gut Microbiota Contributes to Host Defense Against Klebsiella pneumoniae-Induced Liver Abscess
}

\author{
Yahong Zheng' \\ Yuting Ding' \\ Mengran $\mathrm{Xu}^{\prime}$ \\ Haoran Chen' \\ Hui zhang' \\ Yanyan Liu' ${ }^{1-3}$ \\ Weihua Shen ${ }^{4}$ \\ Jiabin $\mathrm{Li}^{1-3,5}$
}

'Department of Infectious Diseases, The First Affiliated Hospital of Anhui Medical University, Hefei, Anhui, People's Republic of China; ${ }^{2}$ Anhui Center for Surveillance of Bacterial Resistance, Hefei, Anhui, People's Republic of China; ${ }^{3}$ Institute of Bacterial Resistance, Anhui Medical University, Hefei, Anhui, People's Republic of China; ${ }^{4}$ Department of Special Clinic, The First Affiliated Hospital of Anhui Medical University, Hefei, Anhui, People's Republic of China; ${ }^{5}$ Department of Infectious Diseases, The Chaohu Affiliated Hospital of Anhui Medical University, Hefei, Anhui, People's Republic of China

\begin{abstract}
Purpose: Klebsiella pneumoniae-induced liver abscess (KLA) is a type of pyogenic liver abscess (PLA), which is a distinct invasive syndrome that has been increasingly reported worldwide over the past two decades. The intestinal microbiota is increasingly recognized as an important modulator that can promote and maintain host immune homeostasis. However, its precise role in liver abscess is unknown. We aimed to investigate the function of the gut microbiota in the host defense against $K$. pneumoniae infection.
\end{abstract}

Methods: We constructed C57BL/6J mice with KLA and analyzed the diversity and richness of the intestinal microflora by $16 \mathrm{~S}$ rRNA sequencing. Next, to create a microbiota-depleted (MD) mouse model, we administered multiple broad-spectrum antibiotics and validated the model using $16 \mathrm{~S}$ rRNA sequencing. At $48 \mathrm{~h}$ after $K$. pneumoniae infection, we assessed the general health condition, liver injury, bacterial loads, and inflammatory factor levels in MD+KLA mice. Additionally, fecal microbiota transplantation (FMT) was conducted in another group of MD+KLA mice prior to K. pneumoniae infection, and we assessed whether the transplantation changed the outcomes.

Results: The diversity of the intestinal flora was significantly changed in KLA mice compared to control mice, with a decrease in beneficial bacteria and an increase in harmful bacteria. The MD+KLA mice exhibited impaired antimicrobial capacity, reduced survival, increased inflammation and liver damage at $48 \mathrm{~h}$ after $K$. pneumoniae infection compared to the KLA mice. However, FMT normalized the inflammatory cytokine levels, reduced liver damage, and increased survival.

Conclusion: This study identified the gut microbiota as a protective factor against $K$. pneumoniae infection. The role of FMT in KLA should be investigated in future clinical studies.

Keywords: Klebsiella pneumoniae, liver abscess, gut microbiome, fecal microbiota transplantation, inflammation

\section{Introduction}

Klebsiella $(K$.$) pneumoniae is a well-known human nosocomial pathogen. Most com-$ munity-acquired $K$. pneumoniae infections involve pneumonia or urinary tract infections. However, over the past two decades, a distinct invasive syndrome known as K. pneumoniae-induced liver abscess (KLA) has been increasingly reported in Asia as a global disease, and it causes high morbidity and mortality. ${ }^{1}$ It is easy to misdiagnose the symptoms or miss them entirely, and difficult to control the development of the disease. The traditional clinical treatment of KLA mainly includes antibiotics and symptomatic management. It is of great importance to explore new effective treatments.

The gut microbiome ( $>1000$ species, with 100 trillion bacteria) is the largest and most diverse microbiome in the host. ${ }^{2}$ As an active participant in the host defense 
system, the intestinal flora supports mucosal immunity and regulates the immune system. ${ }^{3}$ In Streptococcus pneumoniae-induced sepsis, the intestinal microbiota protects against damage by regulating phagocytosis by alveolar macrophages. ${ }^{4}$ Meanwhile, previous studies have confirmed that the intestinal flora has an effect on $K$. pneumoniae colonization. For instance, a healthy gut microbiome provides an extra layer of defense and helps eliminate exogenous bacteria. However, changes in intestinal flora after antibacterial treatment increased the level of available monosaccharides in the intestinal tract and promoted the growth of pathogenic or opportunistic bacteria K. pneumoniae. ${ }^{5}$ More persuasive, we also found that antimicrobial treatment have a pervasive and long-lasting effect on the human gut microbiota in clinical practice, especially in intensive care unit patients receiving antibacterial agents. ${ }^{6,7}$ In vitro studies, gut microbiota metabolites-SCFAs directly inhibited the growth of drug-resistant enterobacteria, including carbapenemase-producing K. pneumoniae. Besides, SCFAs prevented the replication of $K$. pneumoniae by lowering intracellular $\mathrm{pH}^{8}{ }^{8}$ With the increasing understanding of the relationship between the gut microbiome and host immunity against infectious diseases, it is urgent to explore regulating the gut microbiome in order to prevent and treat infections. Recent research showed that the gut microbiota plays an important role in the regulation of mastitis, compared with those in control mice, $S$. aureus-induced mastitis mice were observed Increased blood-milk barrier permeability resulted to increasing abundance of pathogenic Enterobacter bacteria; however, feces microbiota transplantation (FMT) reversed these effects. ${ }^{9}$ Therefore, it is worth exploring whether the intestinal microbiota can reduce liver injury and inflammation, enhancing the ability of the liver to resist the effects of hypervirulent $K$. pneumoniae invasion during the pathogenesis of KLA.

We hypothesized that the intestinal microbiota can enhance the host's defenses against $K$. pneumoniae. In the current study, we assessed the survival, histopathological severity, and serum biochemical and inflammatory indicators in microbiota-depleted (MD) mice infected with K. pneumoniae with or without FMT. The results suggested that the intestinal microbiota plays a protective role in the host defense against $K$. pneumoniae infection. This in-depth study of the relationship between the intestinal microbiota and non-mucosal hepatic immunity provides a new approach for the development of KLA treatments.

\section{Materials and Methods}

\section{Animals and Bacterial Strains}

Wild-type female C57BL/6J mice $(20 \pm 2 \mathrm{~g})$ were purchased from the Experimental Animal Center of Anhui Province (Hefei, China). The animals were housed in a specific-pathogen-free environment, given free access to water and food, and exposed to a $12 \mathrm{~h}$ light/dark cycle. The animal study was approved by the Animal Experimentation Ethics Committee of Anhui Medical University (approval NO. LLSC20190253), and experiments were carried out in strict accordance with the Animal Research: Reporting of In Vivo Experiments (ARRIVE) guidelines (the National Centre for the Replacement, Refinement and Reduction of Animals in Research, London, England) for the care and use of laboratory animals.

K. pneumoniae 181608 (serotype $\mathrm{K} 1$, magA+, rmpA+, aerobactin+) was isolated from patients with KLA and maintained by the Anhui Center for Surveillance of Bacterial Resistance (Hefei, China). The isolate was confirmed by time-of-flight mass spectrometry system (ClinTOF-II, Beijing Yixin Bochuang Biotechnology Co., Ltd.). It was cultured in Luria broth at $37^{\circ} \mathrm{C}$ overnight before use.

\section{KLA Mouse Model}

To create a KLA mouse model (KLA group), mice were administered oral mid-log-phase K. pneumoniae 181608 (0.1 $\mathrm{mL}$ of $10^{4} \mathrm{CFU} / \mathrm{mL}$ suspension) using a 21-gauge feeding needle, as described previously. ${ }^{10}$ The mice that died within 24 hours were excluded from the group. The control group (Con group) was given the same amount of phosphate-buffered saline (PBS) intragastrically. All mice were killed 48 hours after $K$. pneumoniae or PBS administration, and the liver, fecal, and blood were obtained for analysis. To obtain mouse fecal, first sterilize mouse anus with 95\% ethanol, gently press around anus with sterile cotton ball to promote excretion of mouse fecal, and store collected fecal in refrigerator at $-80^{\circ} \mathrm{C}$. To obtain the blood samples and liver tissues, the mice were first anesthetized, the eyeball blood was taken and the mice were killed, the abdominal skin was disinfected after fixation with adhesive tape, and the abdominal cavity was opened. The liver was removed with sterile forceps and scissors and fixed in formalin. To assess survival and construct survival curves, the mice were monitored 4-5 
times daily for reduced movement, shivering, dyspnea, or circling behavior, and these mice were killed.

\section{Microbiota-Depleted (MD) Mouse Model}

To create an MD mouse model, broad-spectrum antibiotics (ampicillin, $1 \mathrm{~g} / \mathrm{L}$; neomycin sulfate, $1 \mathrm{~g} / \mathrm{L}$; metronidazole, $1 \mathrm{~g} / \mathrm{L}$; vancomycin, $0.5 \mathrm{~g} / \mathrm{L}$; Sigma, USA) were added to the drinking water for 3 weeks to eliminate the intestinal flora, as described previously. ${ }^{11}$ During this time, weight and food intake were recorded. At 2 days after antibiotic water was discontinued, to create the MD+KLA group, $K$. pneumoniae was administered.

\section{FMT}

To create the MD+KLA+FMT group, fecal pellets from randomly chosen healthy mice were used to colonize the guts of MD mice 24 hours prior to $K$. pneumoniae infection. ${ }^{12}$ Briefly, several fecal pellets from different healthy mice were suspended together in PBS (1 fecal pellet $/ 1 \mathrm{~mL}$ PBS). Next, $200 \mu \mathrm{L} /$ day of the fecal solution was administered to the mice by oral gavage for 3 days after antibiotic water was discontinued and before K. pneumoniae infection.

\section{I6S rRNA Pyrosequencing}

Microbial DNA was extracted from the feces of the mice using a QIAamp DNA Stool Mini Kit (Qiagen, Germany) according to the manufacturer's protocol. The DNA concentration and purity was monitored on $1 \%$ agarose gels. The DNA was then diluted to $1 \mathrm{ng} / \mu \mathrm{L}$ using sterile water. The V3-V4 region of the bacterial $16 \mathrm{~S}$ rRNA gene was amplified by PCR $\left(95^{\circ} \mathrm{C}\right.$ for 5 min, followed by 25 cycles at $95^{\circ} \mathrm{C}$ for $30 \mathrm{~s}, 60^{\circ} \mathrm{C}$ for $30 \mathrm{~s}$, and $72^{\circ} \mathrm{C}$ for $25 \mathrm{~s}$, and a final extension at $72^{\circ} \mathrm{C}$ for $5 \mathrm{~min}$ ) using primers 338F 5'-ACTCCTACGGGAGGCAGCA-3' and 806R 5 '-GGACTACHVGGGTWTCTAAT- $3^{\prime}{ }^{13}$ The PCR products were then sequenced using an Illumina HiSeq 2500 System at Beijing Genomics Institute (BGI-Shenzhen, China). ${ }^{14}$ Adaptor sequences and low-quality reads were removed. The remaining high-quality clean data were analyzed with UPARSE software, using the UPARSE-OTU and UPARSE-OTUref algorithms. Sequences with $\geq 97 \%$ similarity were assigned to the same OTUs. We picked a representative sequence for each OTU and used the Ribosomal Database Project (RDP) Classifier (http://www. mothur.org/wiki/RDP_reference_files) ${ }^{15}$ to annotate each representative sequence with taxonomic information.
Thereafter, this taxonomic information was used to calculate the relative bacterial abundances at various taxonomic levels.

\section{Luminex Immunoassays}

A mouse multi-factor detection kit (Univ-Biotech Technology Company, Shanghai, China) was taken out and left at room temperature for $30 \mathrm{~min}$, and standard curves were then constructed using the standard substances. The kit was used to detect interleukin (IL)- $1 \beta$, IL-2, IL-4, IL-6, IL-10, IL-17, tumor necrosis factor (TNF)- $\alpha$, interferon (IFN)- $\gamma$, chemokine (CXCL)- 1 and macrophage chemokine protein (MCP)-1. Thereafter, serum samples of mice were added to plates containing the mixed antibody beads and incubated overnight on a shaking table at $4^{\circ} \mathrm{C}$. Next, the samples were washed with the washing solution and a biotin-labeled detection antibody complex was added and incubated at room temperature for $1 \mathrm{~h}$. After washing thoroughly, phycoerythrin (PE)-streptavidin was added and the samples were assessed using a Luminex X-200 System (Invitrogen, CA, USA).

\section{Histology}

Small intestine and liver tissues were fixed in $10 \%$ buffered formalin and processed for hematoxylin and eosin (H\&E) analysis. To assess the liver histological score, the slides were blindly scored for liver inflammation on a scale of $1-8$, as previously described. ${ }^{16}$

\section{Immunohistochemical (IHC) Staining}

For IHC staining of small intestine, the tissues were fixed with $10 \%$ buffered formalin, embedded in paraffin, and then underwent dewaxing and hydration. The sections were then treated with periodate-inactivated enzyme for $1 \mathrm{~min}$ to deactivate endogenous peroxidases. Thereafter, they were microwaved in citrate buffer $(0.01 \mathrm{~mol} / \mathrm{L}, \mathrm{pH}$ $6.0,20 \mathrm{~min}$ ) for antigen retrieval. Then, the sections were treated with $5 \%$ fetal bovine serum albumin in PBS at $37^{\circ} \mathrm{C}$ for $10 \mathrm{~min}$ to minimize non-specific staining.

Small intestine sections were incubated with anti-chromogranin $\mathrm{A}(\mathrm{CgA}$; a marker of intestinal neuroendocrine cells that indirectly reflects intestinal mucosal secretion; 1:100; cat. no. 134972, Abcam, USA), anti-Ki67 (a marker of proliferation and differentiation; 1:100; cat. no. 16667, Abcam, USA), or anti-Muc2 (the main component of the intestinal mucus layer; 1:2000; cat. no. 272692, Abcam, USA) monoclonal antibodies at $4^{\circ} \mathrm{C}$ overnight. The next day, the sections were incubated with a secondary 
antibody (biotin-labeled goat anti-rabbit IgG) for $20 \mathrm{~min}$ at $37^{\circ} \mathrm{C}$, and then treated with a streptavidin-biotin peroxidase complex (Zhongshan Golden Bridge Biotechnology, Beijing, China) at $37^{\circ} \mathrm{C}$ for $30 \mathrm{~min}$. Finally, staining was visualized using diaminobenzidine (ZLI-9018; ZsBio, Beijing, China). The mean optical density of ten randomly selected fields for each slide was used as the relative level of $\mathrm{CgA}, \mathrm{Ki67}$, and Muc2 for statistical analysis.

\section{Quantitative Real-Time Polymerase Chain Reaction (qRT-PCR)}

The total RNA was extracted from liver tissues using TRIzol (TaKaRa Biotechnology Co., Ltd., Dalian, China) and reverse transcribed into cDNA by RevertAid reverse transcriptase (Thermo Fisher Scientific, Rockford, IL, USA). qRT-PCR was performed on a LightCycler 480 Instrument to quantify the mRNAs of inflammatory cytokines and chemokines in the liver. The primers were as follows: IL-1 $\beta$-forward: 5-CATCCAGCTTCAAATCTCGCA-3, IL-1 $\beta$-reverse: 5-GA TGAAGGAAAAGAAGGTGCTC-3; IL-6-forward: 5-ACTT CACAAGTCGGAGGCTTA-3, IL-6-reverse: 5-ATCCA GTTTGGTAGCATCCATC-3; IL-10-forward: 5-CTTACTG ACTGGCATGAGGATCA-3, IL-10-reverse: 5-GCAGCTC TAGGAGCATGTGG; TNF- $\alpha$-forward: 5-ACTGAACTT CGGGGTGATCG-3, TNF- $\alpha$-reverse: 5-TTGAGATCCAT GCCGTTGGC-3; MCP-1-forward: 5-AGTAGGCTGGAG AGCTACAAGA-3, MCP-1-reverse: 5-TGCTGAAGACCTT AGGGCAGAT-3; glyceraldehyde 3-phosphate dehydrogenase (GAPDH)-forward: 5-GTCAAGGCCGAGAATG GGAA-3, GAPDH-reverse: 5-CTCGTGGTTCACACCC ATCA-3.

\section{Biochemical Indicators}

Mouse serum samples were stored at $-80^{\circ} \mathrm{C}$ before assessment. Creatinine $(\mathrm{Cr})$, blood urea nitrogen $(\mathrm{BUN})$, lactic dehydrogenase (LDH), alanine aminotransferase (ALT), and aspartate aminotransferase (AST) were measured with kits purchased from Jiancheng Bioengineering Institute (Nanjing, China).

\section{K. pneumoniae Bacterial Loads}

To quantify the bacterial loads, blood or liver homogenate was aseptically collected, placed in heparin-containing PBS, serially diluted, and then added $(0.1 \mathrm{~mL})$ to Mueller-Hinton Agar plates and incubated at $37^{\circ} \mathrm{C}$ overnight. The number of CFUs of $K$. pneumoniae was then quantified.

\section{Clinical Score}

At 48 hours after $K$. pneumoniae infection, a clinical score was calculated to evaluate the presence and severity of liver abscess in each mouse, based on the following six factors (each scored as 1 or 0 ): periorbital exudate, lethargy, diarrhea, tremors, labored respiration, and piloerection. $^{17}$

\section{Statistical Analysis}

Normally distributed variables are presented as mean \pm SEM. For normally distributed data, two-sample $t$-test was used to evaluate the differences between two groups, and one-way analysis of variance (ANOVA) was used to evaluate the differences among multiple groups. For non-normally distributed data, the two-tailed Mann-Whitney $U$-test was used to evaluate the differences between pairs of groups. To evaluate the differences in the abundances of individual taxa between the groups, STAMP software was utilized. To identify differences in microbial communities between the two groups, analysis of similarities (ANOSIM) was performed based on the Bray-Curtis dissimilarity distance matrices. Kaplan-Meier survival analysis (probability multiplication) was used to assess survival rates. Results were considered statistically significant if $P<0.05$. All graphs were generated using GraphPad Prism 7.

\section{Results}

\section{Experimental Design}

To gain in-depth insights into the role that the intestinal microbiota plays during KLA, MD mice were established by administering broad-spectrum antibiotics (ampicillin, neomycin, metronidazole, and vancomycin) via the drinking water of wild-type mice for 3 weeks (Figure 1A). At the early stage of $\mathrm{MD}$, due to the antibiotic odor, the mice ate less and lost weight. After 5-6 days of adaptation, there was no difference in the body weight or food intake between MD and Con mice (Figures 1B and C).

To assess whether the antibiotic treatment effectively established MD mice, we subjected the DNA from the feces of the mice to $16 \mathrm{~S}$ rRNA sequencing, and then determined the abundances and diversity of the intestinal flora. Figure 1D shows the rarefaction curve (with the number of OTUs plotted against the number of individuals), which indicates the adequacy of the sample size and estimates species richness. As the number of sampled individuals increases, the curve plateaus, indicating that the sample size 


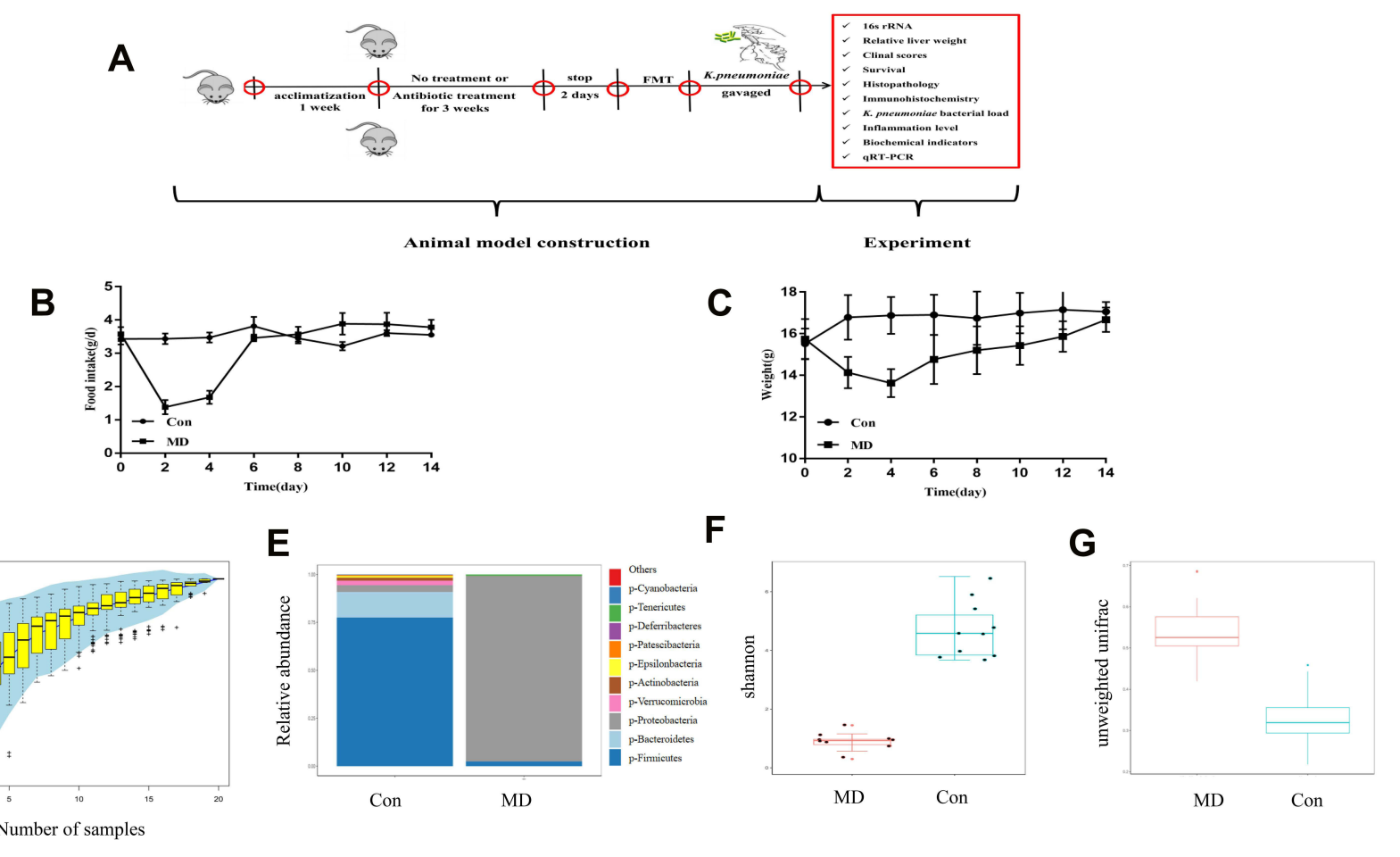

Figure I Experimental design. (A) Wild-type mice were treated for 3 weeks with broad-spectrum antibiotics (ampicillin, neomycin, metronidazole, and vancomycin) in their drinking water to create microbiota-depleted (MD) mice. The mice underwent FMT of normal flora from healthy mice before challenge with $K$. pneumoniae. Subsequently, body weight, survival, bacterial load, and inflammatory factor levels were determined at various time points after $K$. pneumoniae infection. Food intake (B) and weight (C) of MD and healthy control (Con) mice. (D) Rarefaction curve showing that the sample size was sufficient in a boxplot of the observed species. $\mathrm{X}$ is sample size. $\mathrm{Y}$ is number of OTUs. (E) Bar chart of relative abundances of intestinal bacteria in MD and Con mice at phylum level. $\alpha$-diversity (Shannon index) (F) and $\beta$-diversity (ANOSIM) (G) based on I6S rRNA genes in MD and Con mice. $\mathrm{P}<0.00 \mathrm{I}$ (two-tailed Mann-Whitney U-test).

met or even exceeded the experimental requirements. As expected, the MD mice exhibited a sharp decline in relative abundances at the phylum level (Figure 1E). Additionally, $\alpha$ and $\beta$-diversity analyses showed significant differences in flora composition between the $\mathrm{MD}$ and Con mice $(P<0.001)$ (Figures $1 \mathrm{~F}$ and $\mathrm{G})$. These data strongly demonstrated that the MD mice could be used as a substitute for a sterile mouse model in subsequent experiments.

To assess the effects of FMT from healthy mice on KLA, MD mice underwent $K$. pneumoniae challenge $\left(10^{4}\right.$ $\mathrm{CFU} / \mathrm{mL}$ ) to create the MD+KLA mouse model. Prior to this, half of MD+KLA mice were subjected to FMT, while the remaining mice were treated with PBS (still called MD +KLA group). Feces samples were collected at different time points after the $K$. pneumoniae challenge (Figure 1A).

\section{Mice Did Not Have Impaired Intestinal Mucosa Barrier Function}

The protein expression levels of $\mathrm{CgA}$ (a marker of intestinal neuroendocrine cells that indirectly reflects intestinal mucosal secretion), Ki67 (used to evaluate intestinal crypt cell proliferation and differentiation), and Muc2 (the main component of the intestinal mucus layer) are correlated with intestinal function or epithelial integrity. Immunohistochemical staining and observation at high magnification showed that the cytoplasm of CgA-positive cells of livers

was brown, and they were individually scattered or distributed in groups in the mucosa and submucosa. There was no significant difference in the number of CgA-positive cells between the $\mathrm{MD}$ and Con mice $(\mathrm{t}=1.393, P=0.18$; Figures $2 \mathrm{~B}$ and $\mathrm{E})$. There was also no significant difference in the number of Ki67-positive cells between the MD and Con mice based on light microscopy (200x; $\mathrm{t}=-1.658, P=0.115$; Figures $2 \mathrm{C}$ and $\mathrm{F}$ ). Lastly, Muc2 expression in the intestinal mucosa did not differ between the $\mathrm{MD}$ and Con mice $(\mathrm{t}=0.53, P=0.603$; Figures 2D and G).

In addition, $\mathrm{H} \& \mathrm{E}$ staining in both the $\mathrm{MD}$ and Con groups showed normal intestinal epithelial structure and clear stratification, no edema or congestion in the mucosal muscular layer, no reduction in the number of goblet cells, and no inflammatory cell infiltration (Figure 2A). Thus, the broad-spectrum antibiotics did not influence the gut architecture or epithelial integrity. 

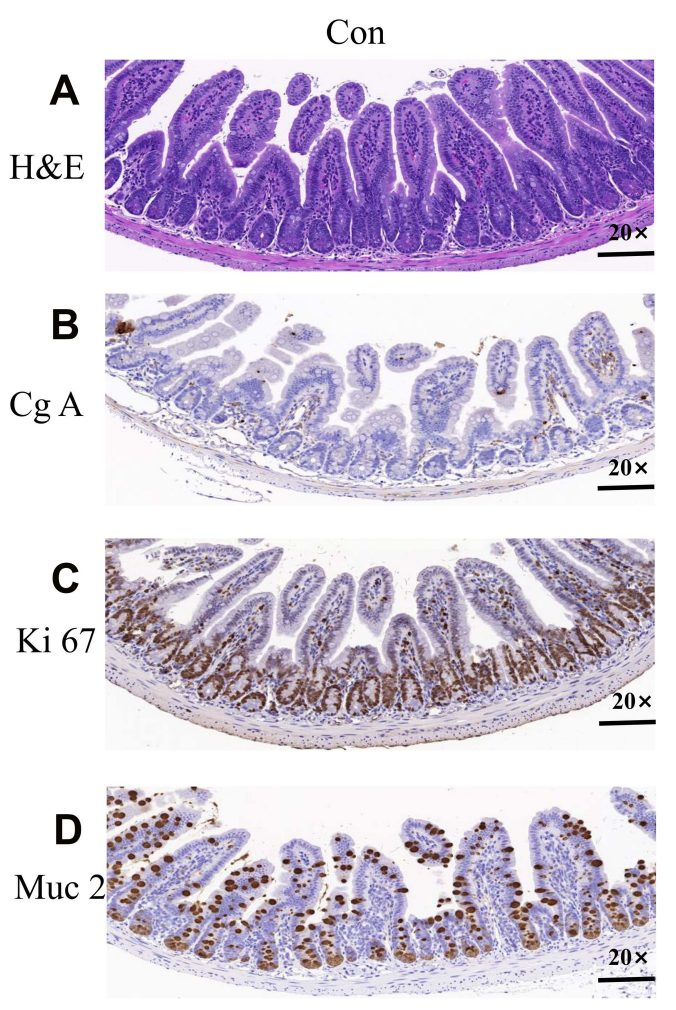
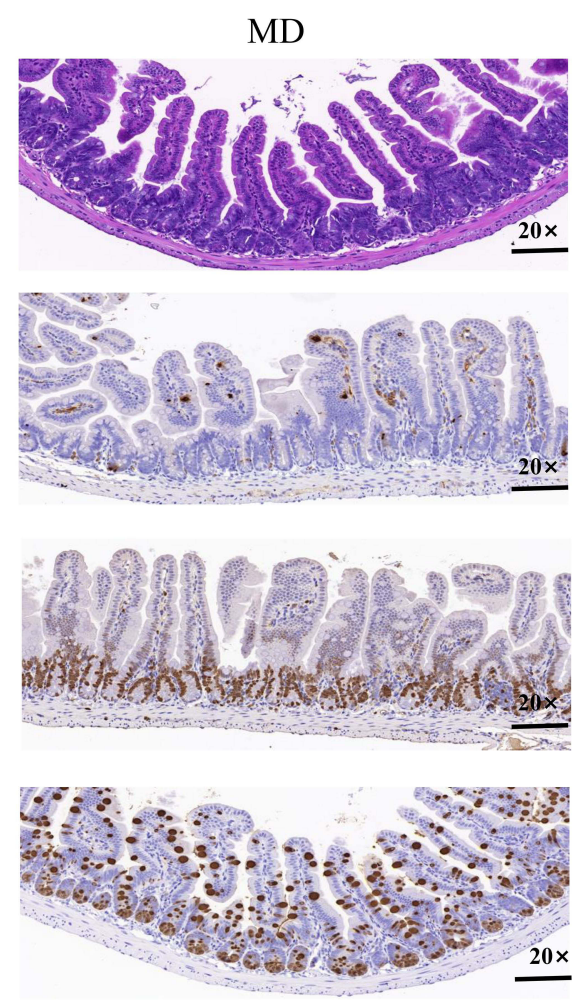
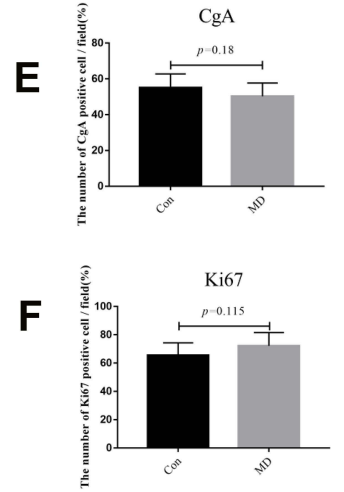

G

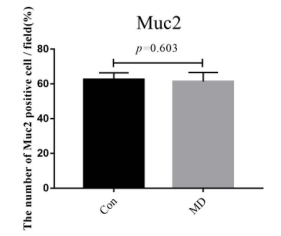

Figure 2 MD mice did not have impaired intestinal mucosa barrier function. Small intestine sections of healthy control (Con; left) and MD (right) mice were stained with hematoxylin and eosin $(\mathrm{H} \& \mathrm{E})$ and observed under a microscope (magnification, $\times 200)$. Representative immunohistochemical staining of chromogranin $\mathrm{A}(\mathrm{H} \& \mathrm{E})(\mathbf{A})(\mathrm{CgA})$ (B), Ki67 (C), and Muc2 (D) in small intestine sections from MD and Con mice (magnification, $\times 200$ ). Number of cells that were positive for CgA (E), Ki67 (F), and Muc2 (G) in MD and Con mice ( $n=10 /$ group). Cells in ten high-power fields were counted. Data are expressed as mean \pm SEM.

\section{Alterations of the Gut Microbiome in KLA Mice Compared to Con Mice}

The V3-V4 region of the bacterial 16S rRNA gene was amplified by PCR, the microbial community diversity and composition were compared between KLA and Con mice. KLA had no effect on the OTU richness (Figure 3A) or Shannon index (Figure 3B). However, ANOSIM ( $\beta$-diversity), a nonparametric method for analyzing the similarity between high-dimensional data sets, indicated that the microbiota composition in the KLA mice was altered compared to that in the Con mice (Figure 3C). Thus, the results revealed that serotype K1 $K$. pneumoniae infection changed the microbiota composition, but there was no substantial difference in abundance compared to the Con group.

Thereafter, OTUs annotated with taxonomic information were used to compare the two groups at the phylum and genus levels. The dominant phyla in both groups were Firmicutes, Bacteroidetes, Proteobacteria, Verrucoidetes, and Actinobacteria (Figure 3D), with Firmicutes plus Bacteroidetes accounting for $>75 \%$ of all bacteria in both groups. The Firmicutes/Bacteroidetes ratio was decreased in the KLA group compared to the
Con group (Figure 3D). In addition, KLA mice had a higher abundance of Proteobacteria and a lower abundance of Actinobacteria ( $<5 \%$ of the total) at the phylum level $(P<0.05$; Figure 3E). At the genus level, L. reuteri was the dominant bacteria in both groups, but it was significantly reduced in the KLA mice compared to the Con mice $(P<0.05$; Figure 3F). The KLA mice had high abundances of Oscillospira and Escherichia, and a lower abundance of Desulfovibrio ( $P<0.05$; Figure $3 \mathrm{G})$. On the basis of these data, we concluded that the disordered gut flora is related to the pathogenesis of KLA. In addition to the final analysis of the intestinal flora of mice in the KLA and CON groups, we further analyzed and screened specific taxa that may have biological markers. We performed a linear discriminant analysis effect size (LEfSe) analysis to identify specific taxa that varied in abundance and that would potentially be used as biomarkers. In total, 40 genera were identified with LDA scores $>2$ (Figure S1). Figure S2 is a Cladogram, which can more vividly display the microbiota (biomarker) with important roles from phylum, class, order, family and genus levels between two groups. 

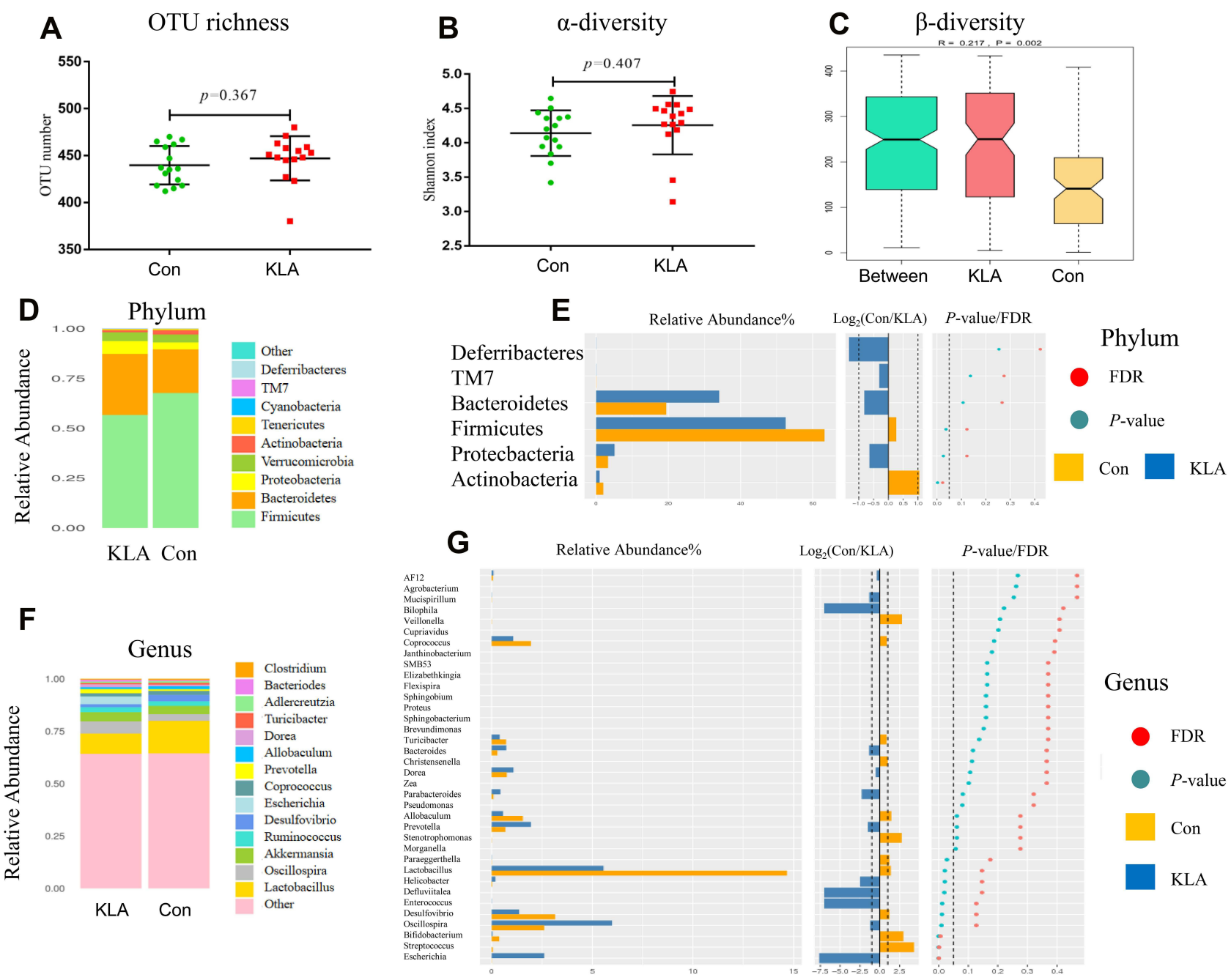

Figure 3 Alterations in the gut microbiome in KLA mice. OTU richness (A) and Shannon index (B) based on I6S rRNA genes from KLA and Con mice. Each point represents a mouse ( $n=15$ /group). (C) OTU analysis of similarities (ANOSIM) illustrates the gut microbiome $\beta$-diversity ( $n=15 / g r o u p)$. Relative abundances (percentage of total sequences) of gut bacteria at the phylum (D) and genus (F) levels. Species with abundance $<0.5 \%$ in all samples were merged together into "Others". Differences between KLA and Con mice at the phylum (E) and genus (G) levels, based on Mann-Whitney U-tests.

\section{Gut Microbiota Protects Against Liver Damage and Death During KLA}

To determine the protective role of the gut microbiota against hepatic bacterial infection, we semi-quantitatively scored liver histology slides in the KLA, MD+KLA, and $\mathrm{MD}+\mathrm{KLA}+\mathrm{FMT}$ groups after $K$. pneumoniae challenge. All $K$. pneumoniae-infected mice had severe KLA at 48 $\mathrm{h}$ post infection. However, the MD+KLA mice had the largest and most severe abscesses, with vacuolar degeneration, cytoplasmic necrosis of hepatocytes, and extensive inflammatory cell infiltration (Figure 4A). The histological score, which directly reflects hepatic damage, was highest in the MD+KLA mice, but was reduced by FMT (Figure 4B).
Additionally, MD+KLA mice had a significantly increased mortality rate within 7 days following K. pneumoniae infection compared to KLA mice (Figure 4C). Remarkably, FMT significantly improved the survival rate (Figure $4 \mathrm{C}$ ). At $48 \mathrm{~h}$ after $K$. pneumoniae infection, MD+KLA mice had increased hepatic and blood bacterial loads compared to the KLA and MD+KLA +FMT mice (Figures 4F and G). MD+KLA+FMT mice had a lower blood (but not hepatic) bacteria load than MD+KLA mice (Figures 4F and $\mathrm{G}$ ). As expected, the relative liver weight $(P=0.028)$ and clinical score $(P=0.019)$ (which indicate the general condition of the mice) were increased in MD+KLA mice compared to KLA mice, and FMT only reduced the latter score $(P=0.000)$ compared to that in $\mathrm{MD}$ + KLA mice (Figures 4D and E). 

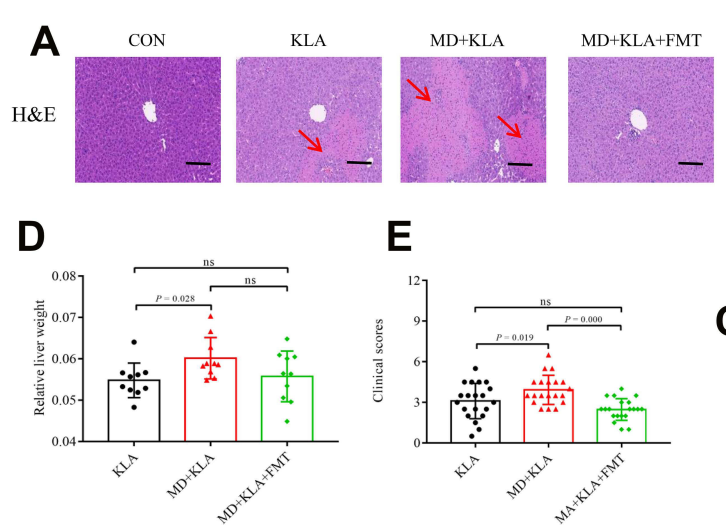

E
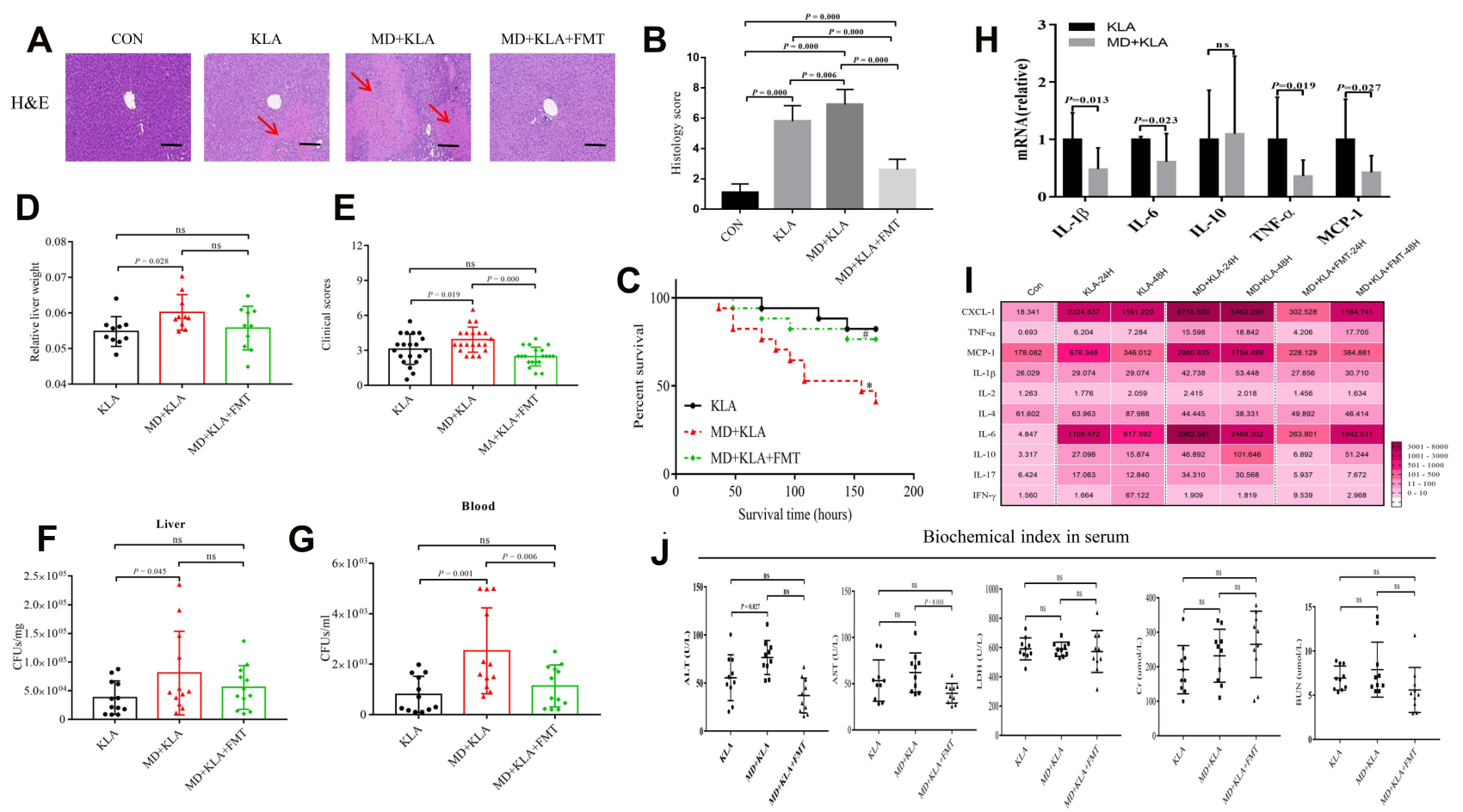

Figure 4 Gut microbiota protects against organ damage during KLA. (A) Representative liver sections of H\&E staining at $48 \mathrm{~h}$ after $\mathrm{K}$. pneumoniae infection in the KLA, MD $+K L A$, and MD+KLA+FMT groups (magnification, 200x). Red arrowheads indicate KLA. (B) Histological scores indicating evidence of liver abscess and necrosis ( $\mathrm{n}=10 /$ group). (C) Kaplan-Meier survival curves of KLA, MD+KLA, MD+KLA+FMT groups after $K$. pneumoniae infection ( $n=10 /$ group). Relative liver weight ( $n=10 /$ group) (D) and clinical score ( $n=20$ /group) (E) were used to evaluate the health status of the mice. K. pneumoniae load (CFUs) in liver (F) and blood (G) at $48 \mathrm{~h}$ after infection ( $\mathrm{n}=12 / \mathrm{group})$. (H) IL-I $\beta$, IL-6, IL-I0, TNF- $\alpha$, and MCP-I mRNA expression in liver tissues based on qRT-PCR ( $\mathrm{n}=10 /$ group). (I) Luminex heatmap of serum inflammatory cytokine and chemokine levels at 24 and $48 \mathrm{~h}$ after infection ( $\mathrm{n}=10$ /group). (J) Serum ALT, AST, LDH, Cr, and BUN levels at $48 \mathrm{~h}$ after infection ( $\mathrm{n}=10 / \mathrm{group})$. Data are presented as mean \pm SEM. $* P<0.05$ vs KLA; ${ }^{\#} P<0.05$ vs MD+KLA.

Abbreviation: n.s., not significant.

As the inflammatory response related to combating pathogens can lead to organ damage, we measured the serum levels of inflammatory cytokines and chemokines. IL-1 $\beta, \quad$ IL-6, IL-10, IL-17, TNF- $\alpha, \quad$ MCP-1, and $\mathrm{C}-\mathrm{X}-\mathrm{C}$ motif chemokine (CXCL)-1 were increased in MD+KLA mice after $K$. pneumoniae infection compared to KLA mice (Figure 4I). Additionally, qRT-PCR showed that hepatic IL-0.1 $\beta$, IL-6, TNF- $\alpha$, and MCP-1 mRNA levels were increased in MD+KLA mice compared to KLA mice (Figure 4H). Regarding the biochemical indicators of liver injury, the MD+KLA mice had an elevated plasma AST level but BUN and $\mathrm{Cr}$ were not affected compared to KLA mice (Figure 4J). In brief, these results suggested that the gut microbiota protects against organ injury during KLA.

\section{Discussion}

PLA is a life-threatening condition that involves bacterial infection of the hepatic parenchyma. It is frequently observed worldwide, ${ }^{18}$ but has remarkably increased in Asia. For example, in Taiwan, the annual incidence gradually increased from 10.83 to 15.45 cases per 100,000 from 2000 to 2011, and the mortality rate was up to $19 \% .{ }^{19} \mathrm{~K}$. pneumoniae has become a dominant causal agent, far surpassing E. coli, accounting for $50-88 \%$ of PLA patients in Asian countries, the United States, and Europe, and it tended to spread globally. ${ }^{20}$ Importantly, K. pneumoniae from KLA cases is generally hypervirulent and can migrate from the primary infection site to distant sites, thereby leading to extrahepatic complications, such as lung abscess, endophthalmitis, meningitis, and necrotizing fasciitis. ${ }^{21}$ Lack of typical liver abscess symptoms and poor awareness of treatment strategies have been reported to be important reasons for delayed treatment. ${ }^{22}$ Routine prophylactic antibiotic use has recently accelerated the emergence of resistance among the hypervirulent K. pneumoniae. Therefore, it is urgent to develop effective new treatments. ${ }^{23}$

Recently, research breakthroughs regarding the gut microbiome in healthy and diseased states have made a considerable impact on the management of infectious diseases. ${ }^{24}$ In this study, we made considerable progress 
regarding understanding the relationship between the intestinal flora and KLA. First, K. pneumoniae infection significantly altered the intestinal microbial structure, including profoundly changing the abundances of the dominant phyla such as Proteobacteria. At this point, it is not difficult to draw the conclusion that dysbiosis might be one of the reasons for KLA progression. Second, we found that antibiotic treatment prior to $K$. pneumoniae infection (in MD+KLA mice) weakened the protective effect of the intestinal flora, as evidenced by increased disease severity, reduced survival, increased tissue bacterial load, and high levels of inflammatory factors. More importantly, FMT from healthy mice into MD mice, 24 hours before the $K$. pneumoniae challenge, normalized these indicators. Taken together, these findings indicate that the regulation of the intestinal flora is a promising anti-infective treatment strategy for KLA.

Maintaining a good symbiotic relationship between the host and intestinal microorganisms is crucial to human health. ${ }^{25}$ A variety of acute and chronic diseases, including metabolic diseases, ${ }^{26}$ cardio-cerebrovascular diseases, ${ }^{27,28}$ inflammatory diseases, ${ }^{29}$ and respiratory diseases, ${ }^{30}$ are closely related to disordered intestinal flora. We found that there was no significant difference in OTU richness and Shannon index, but there was a profound change in the gut microbiota composition between the KLA and Con groups. Firmicutes and Bacteroidetes, as the dominant phyla in the gut, accounted for $>75 \%$ of all bacteria in both the KLA and Con groups. K. pneumoniae infection increased the abundance of Proteobacteria, part of which can be harmful, disrupting the microbial balance in the intestine. It has been reported that Proteobacteria (including the pathogenic bacteria E. coli, Salmonella, and Vibrio cholerae) is maintained at a lower level in the healthy host intestine compared to in certain diseased states. Many proliferating pathogens produce proinflammatory factors that cause intestinal inflammation, leading to changes in Proteobacteria in the gut. ${ }^{31,32}$ The decreased beneficial bacteria and enhanced harmful bacteria damaged the intestinal barrier and increased intestinal permeability, leading to a large $K$. pneumoniae influx into the portal vein and liver. $K$. pneumoniae eventually formed liver abscesses through infection and colonization. In summary, our findings fill in the gap regarding the relationship between the gut microbiota and KLA.

To extensively explore the beneficial role of the intestinal flora in liver infection, we constructed an antibiotictreated MD mouse model. Adding multiple broad-spectrum antibiotics to drinking water has been reported to be sufficient to eliminate intestinal flora of the mice. ${ }^{11}$ We used $16 \mathrm{~S}$ rRNA sequencing to verify the successful construction of the model. As expected, compared to the Con group, the relative bacterial abundances in the MD mice were sharply reduced, and there were huge differences in gut flora composition. In addition, the intestinal histopathology showed that the antibiotics had no effect on the intestinal mucosal barrier function which is the guarantee to prevent the translocation and spread of intestinal bacteria. In a word, the MD mice could be used to replace a sterile mouse model for the subsequent experiments. Disruptions in the gut flora may exacerbate the progression of some diseases. For instance, the absence of intestinal flora can aggravate bacterial infection induced lung damage, ${ }^{33,34}$ but this is rarely related to KLA. We found that during $K$. pneumoniae challenge, the protective effects of the intestinal flora against bacterial infection involved preventing organ injury, clinical symptoms, and bacterial transmission. Semi-quantitative analysis of liver histopathology indicated evidence of liver abscess in all K. pneumoniae-infected mice, which was consistent with the results of Lin et al. ${ }^{16}$ Furthermore, the MD+KLA mice had a higher histopathological score than KLA mice, with more inflammatory cell infiltration and extensive liver cell necrosis.

Regarding the clinical symptom scores (which were used to assess the infection severity and are proportional to the level of symptom deterioration ${ }^{17}$ ), MD+KLA mice, without the help of their gut microbiota, developed purulent discharge from the corners of the eyes, lethargy, diarrhea, tremors, and piloerection after $K$. pneumoniae infection. The aggravated symptoms and high mortality rate clearly emphasized the importance of the gut flora. In addition, consistent with the severity of liver injury observed, the MD+KLA mice had higher hepatic and blood bacterial loads compared to the KLA mice.

Moreover, the pathogenic bacteria invasion and inflammatory factor infiltration increased the relative liver weight in MD+KLA mice compared to KLA mice. During $K$. pneumoniae infection, MD+KLA was shown to increase the degree of systemic inflammatory response, particularly regarding the levels of proinflammatory factors and chemokines. Serum IL-1 $\beta$, IL-6, IL-10, IL-17, TNF- $\alpha$, MCP-1, and CXCL-1 levels were increased in $\mathrm{MD}+\mathrm{KLA}$ mice compared to KLA mice after $K$. pneumoniae challenge. Moreover, qRT-PCR also showed that the mRNA levels of IL-1 $\beta$, IL-6, TNF- $\alpha$, and $\mathrm{MCP}-1$ in the liver were higher in the MD+KLA 
mice. Clinically, ALT, AST, and LDH are commonly used to evaluate liver function, ${ }^{35}$ and they are elevated in liver abscess patients. In this study, ALT was significantly elevated in KLA mice, and the difference was even more pronounced in MD+KLA mice. Taken together, these findings support the concept that a lack of gut flora weakens the host's gut defenses.

It is urgent to identify interventions to prevent or treat KLA. FMT, in which the feces of a healthy individual (containing the normal flora in the gut of human) are transplanted into patients' intestines, has been used since the 1950s. ${ }^{36,37}$ Increasing evidence demonstrates that FMT, which is a new and underexplored method to alter the gut microbiota, can treat chronic gastrointestinal infections, inflammatory bowel diseases, and sepsis. ${ }^{38}$ In addition to treating bowel-related diseases, the bacteria involved in FMT (such as Bacteroidetes) can proliferate in hosts with systemic pathogen infection and produce butyrate to protect against this infection. ${ }^{39}$ In the current study, the survival curves indicated that FMT increased the survival of MD+KLA mice. FMT also normalized serum CXCL-1, TNF- $\alpha$, MCP-1, IL-1 $\beta$, IL-6, and IL-17 in MD + KLA mice. Expectedly, FMT decreased ALT, which is correlated with liver injury. However, FMT did not affect hepatic bacterial loads at $48 \mathrm{~h}$ after $K$. pneumoniae infection. In general, these findings suggested that intestinal flora reduces the negative effects of the host's reaction against $K$. pneumoniae infection.

\section{Conclusion}

Our study suggested that dysbiosis gut microbiota may be related to the pathogenesis of KLA. However, 16s rRNA sequencing does not provide sufficient resolution, and the broad pattern of change is not impressive. Metagenomic techniques will be used to accurately identify the origin of species and obtain "high resolution" fine composition spectra of species or below the species level in the future. In addition, the model strains were only derived from serotype $\mathrm{K} 1 \mathrm{~K}$. pneumoniae, leading to certain limitations of the research results. It is imperative to explore more serotypes of $K$. pneumoniae, $S$. aureus, $E$. coli and anaerobic bacteria. Gut microbiota as a protective factor against $K$. pneumoniae infection, which may be a new therapeutic strategy for KLA patients. But they need to be confirmed in extensive clinical research. Caution is needed when extrapolating data from animal experiments to human application, which involves more factors that cannot be controlled for, including disease complications, drug interference, and genomic mutations. In the future, we intend to explore the specific metabolites, targets, and downstream signaling pathways affected by the intestinal flora involved in host immune regulation.

\section{Acknowledgments}

We are extremely thankful to Yuanyuan Dai and Jiajia Li for their technical support.

\section{Funding}

This study was supported by the National Natural Science Foundation of China (no. 81973983), National Science and Technology Major Project (no. 2017ZX10204401), Borrowing and Transferring Subsidy Project in 2019, Hefei (no. J2019Y04), Collaborative Tackling and Public Health Collaborative Innovation Project in Anhui Province (no. GXXT-2020-018), Joint Construction Project of Clinical Medicine University and Hospital (no. 2021lcxk006), and Natural Science Research Project of Universities in Anhui Province (no. KJ2020A0176). The sponsors had no role in any of the stages from study design to submission of the manuscript for publication.

\section{Disclosure}

The authors report no conflicts of interest in this work.

\section{References}

1. Siu LK, Yeh KM, Lin JC, et al. Klebsiella pneumoniae liver abscess: a new invasive syndrome. Lancet Infect Dis. 2012;12(11):881-887. doi:10.1016/S1473-3099(12)70205-0

2. Tan GSE, Tay HL, Tan SH, et al. Gut microbiota modulation: implications for infection control and antimicrobial stewardship. Adv Ther. 2020;37(10):4054-4067. doi:10.1007/s12325-020-01458-z

3. Caballero S, Pamer EG. Microbiota-mediated inflammation and antimicrobial defense in the intestine. Annu Rev Immunol. 2015;33 (1):227-256. doi:10.1146/annurev-immunol-032713-120238

4. Schuijt TJ, Lankelma JM, Scicluna BP, et al. The gut microbiota plays a protective role in the host defence against pneumococcal pneumonia. Gut. 2016;65(4):575-583. doi:10.1136/gutjnl-2015-309728

5. Ng KM, Ferreyra JA, Higginbottom SK, et al. Microbiota-liberated host sugars facilitate post-antibiotic expansion of enteric pathogens. Nature. 2013;502(7469):96-99. doi:10.1038/nature12503

6. Schuijt TJ, van der Poll T, de Vos WM, et al. The intestinal microbiota and host immune interactions in the critically ill. Trends Microbiol. 2013;21(5):221-229. doi:10.1016/j.tim.2013.02.001

7. Dethlefsen L, Relman DA. Incomplete recovery and individualized responses of the human distal gut microbiota to repeated antibiotic perturbation. Proc Natl Acad Sci U S A. 2011;108 Suppl 1 (Supp11):4554-4561. doi:10.1073/pnas.1000087107

8. Sorbara MT, Dubin K, Littmann ER, et al. Inhibiting antibiotic-resistant Enterobacteriaceae by microbiota-mediated intracellular acidification. $J$ Exp Med. 2019;216(1):84-98. doi:10.1084/ jem.20181639 
9. Hu X, Guo J, Zhao C, et al. The gut microbiota contributes to the development of Staphylococcus aureus-induced mastitis in mice. ISME J. 2020;14(7):1897-1910. doi:10.1038/s41396-020-0651-1

10. Chen N, Wang LL, Xue J, et al. Different metabolic profiles of K1 serotype and non-serotype K1 and K2 Klebsiella pneumoniae isolates in oral infection mice model. Microb Pathog. 2014;75:41-48. doi:10.1016/j.micpath.2014.08.006

11. Clarke TB, Davis KM, Lysenko ES, Zhou AY, Yu Y, Weiser JN. Recognition of peptidoglycan from the microbiota by Nod1 enhances systemic innate immunity. Nat Med. 2010;16(2):228-231. doi: $10.1038 / \mathrm{nm} .2087$

12. Ubeda C, Bucci V, Caballero S, et al. Intestinal microbiota containing barnesiella species cures vancomycin-resistant Enterococcus faecium colonization. Infect Immun. 2013;81(3):965-973. doi:10.1128/ IAI.01197-12

13. Caporaso JG, Lauber CL, Walters WA, et al. Ultra-high-throughput microbial community analysis on the illumina HiSeq and MiSeq platforms. ISME J. 2012;6(8):1621-1624. doi:10.1038/ismej.2012.8

14. Cassir N, Benamar S, Croce O, La Scola B. Clostridium species identification by $16 \mathrm{~S}$ rRNA pyrosequencing metagenomics. Clin Infect Dis. 2016;62(12):1616-1618. doi:10.1093/cid/ciw203

15. Liu R, Hong J, Xu X, et al. Gut microbiome and serum metabolome alterations in obesity and after weight-loss intervention. Nat Med. 2017;23(7):859-868. doi:10.1038/nm.4358

16. Lin YT, Tseng KY, Yeh YC, Yang FC, Fung CP, Chen NJ. TREM-1 promotes survival during Klebsiella pneumoniae liver abscess in mice. Infect Immun. 2014;82(3):1335-1342. doi:10.1128/IAI.01347-13

17. Zhang YF, Sun CC, Duan JX, et al. A COX-2/sEH dual inhibitor PTUPB ameliorates cecal ligation and puncture-induced sepsis in mice via anti-inflammation and anti-oxidative stress. Biomed Pharmacother. 2020;126:109907. doi:10.1016/j.biopha.2020.109907

18. Akhondi H, Sabih DE. Liver abscess. In: StatPearls. Treasure Island (FL): StatPearls Publishing; April 7, 2021.

19. Chen YC, Lin CH, Chang SN, Shi ZY. Epidemiology and clinical outcome of pyogenic liver abscess: an analysis from the National health insurance research database of Taiwan, 2000-2011. J Microbiol Immunol Infect. 2016;49(5):646-653. doi:10.1016/j. jmii.2014.08.028

20. Liu Y, Wang J-Y, Jiang W. An increasing prominent disease of Klebsiella pneumoniae liver abscess: etiology, diagnosis, and treatment. Gastroenterol Res Pract. 2013;2013:258514. doi:10.1155/2013/258514

21. Jun JB. Klebsiella pneumoniae liver abscess. Infect Chemother. 2018;50(3):210-218. doi:10.3947/ic.2018.50.3.210

22. Zhang S, Zhang X, Wu Q, et al. Clinical, microbiological, and molecular epidemiological characteristics of Klebsiella pneumoniae-induced pyogenic liver abscess in southeastern China. Antimicrob Resist Infect Control. 2019;8:166. doi:10.1186/s13756-019-0615-2

23. Shi SH, Zhai ZL, Zheng SS. Pyogenic liver abscess of biliary origin: the existing problems and their strategies. Semin Liver Dis. 2018;38 (3):270-283. doi:10.1055/s-0038-1661363

24. Trompette A, Gollwitzer ES, Yadava K, et al. Gut microbiota metabolism of dietary fiber influences allergic airway disease and hematopoiesis. Nat Med. 2014;20(2):159-166. doi:10.1038/nm.3444
25. Zhao L. The gut microbiota and obesity: from correlation to causality Nat Rev Microbiol. 2013;11(9):639-647. doi:10.1038/nrmicro3089

26. Ma Q, Li Y, Li P, et al. Research progress in the relationship between type 2 diabetes mellitus and intestinal flora. Biomed Pharmacother. 2019;117:109138. doi:10.1016/j.biopha.2019.109138

27. Jin $M$, Qian Z, Yin $J$, et al. The role of intestinal microbiota in cardiovascular disease. J Cell Mol Med. 2019;23(4):2343-2350. doi:10.1111/jcmm.14195

28. Angelucci F, Cechova K, Amlerova J, et al. Antibiotics, gut microbiota, and Alzheimer's disease. J Neuroinflammation. 2019;16 (1):108. doi:10.1186/s12974-019-1494-4

29. Chu H, Tao X, Sun Z, et al. Galactooligosaccharides protects against DSS-induced murine colitis through regulating intestinal flora and

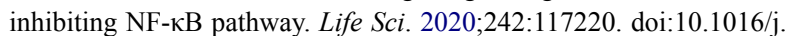
Ifs.2019.117220

30. Felix KM, Jaimez IA, Nguyen TV, et al. Gut microbiota contributes to resistance against Pneumococcal pneumonia in immunodeficient rag-/- mice. Front Cell Infect Microbiol. 2018;8:118. doi:10.3389/ fcimb.2018.00118

31. Shin NR, Whon TW, Bae JW. Proteobacteria: microbial signature of dysbiosis in gut microbiota. Trends Biotechnol. 2015;33(9):496-503. doi:10.1016/j.tibtech.2015.06.011

32. Durbán A, Abellán JJ, Jiménez-Hernández $\mathrm{N}$, et al. Instability of the faecal microbiota in diarrhoea-predominant irritable bowel syndrome. FEMS Microbiol Ecol. 2013;86(3):581-589. doi:10.1111/15746941.12184

33. Budden KF, Gellatly SL, Wood DL, et al. Emerging pathogenic links between microbiota and the gut-lung axis. Nat Rev Microbiol. 2017;15(1):55-63. doi:10.1038/nrmicro.2016.142

34. Lankelma JM, Birnie E, Weehuizen TAF, et al. The gut microbiota as a modulator of innate immunity during melioidosis. PLoS Negl Trop Dis. 2017;11(4):e0005548. doi:10.1371/journal.pntd.0005548

35. Khan MZ, Tahir D, Kichloo A, et al. Pyogenic liver abscess and sepsis caused by Streptococcus constellatus in the immunocompetent host. Cureus. 2020;12(8):e9802. doi:10.7759/cureus.9802

36. El Feghaly RE, Bangar H, Haslam DB. The molecular basis of Clostridium difficile disease and host response. Curr Opin Gastroenterol. 2015;31(1):24-29. doi:10.1097/MOG.000000000000 0131

37. Eiseman B, Silen W, Bascom GS, et al. Fecal enema as an adjunct in the treatment of pseudomembranous enterocolitis. Surgery. 1958;44 (5):854-859.

38. Zhou D, Pan Q, Shen F, et al. Total fecal microbiota transplantation alleviates high-fat diet-induced steatohepatitis in mice via beneficial regulation of gut microbiota. Sci Rep. 2017;7(1):1529. doi:10.1038/ s41598-017-01751-y

39. Kim SM, DeFazio JR, Hyoju SK, et al. Fecal microbiota transplant rescues mice from human pathogen mediated sepsis by restoring systemic immunity. Nat Commun. 2020;11(1):2354. doi:10.1038/ s41467-020-15545-w
Journal of Inflammation Research

\section{Publish your work in this journal}

The Journal of Inflammation Research is an international, peerreviewed open-access journal that welcomes laboratory and clinical findings on the molecular basis, cell biology and pharmacology of inflammation including original research, reviews, symposium reports, hypothesis formation and commentaries on: acute/chronic inflammation; mediators of inflammation; cellular processes; molecular mechanisms; pharmacology and novel anti-inflammatory drugs; clinical conditions involving inflammation. The manuscript management system is completely online and includes a very quick and fair peerreview system. Visit http://www.dovepress.com/testimonials.php to read real quotes from published authors. 